\title{
Lineal illuminating system for automatic $x-y$ graphic colorimetric and texture testing
}

\author{
José A. Gómez Pedrero, Eusebio Bernabeu, and José A. Peñaranda
}

\begin{abstract}
A lineal illuminating system based on an optical fiber array is studied. It can be applied to automatic industrial inspection systems, e.g., those used for chromatic classification of ceramic floor tiles. Improvement in the existing system's performance is achieved by using a collimated beam, as shown by both photometric analysis and experimental results. This system has been implemented as part of a realtime controlling tool in a production line. (C) 1998 Optical Society of America

OCIS codes: $150.2950,040.1240$.
\end{abstract}

\section{Introduction}

Nowadays the development of automatic industrial inspection systems is an important field of applied research. In this paper we analyze the behavior of an apparatus for classification of ceramic floor tiles as soon as they are taken from the baking oven (although it can be applied for classifying other products with planar surfaces such as steel or wood sheets). The classification criteria that we employed group the ceramic tiles that present the same visual appearance to the human eye. We say that two ceramic floor tiles have the same visual appearance if their color and visual texture (we define visual texture below) are the same.

According to these classification criteria, the color image of the test surface must be analyzed to obtain information relative to its color and visual texture. (In general, we speak of the test surface because the inspection machine studied in this paper could be used to analyze any planar surface.) This image must be taken when the test surface is removed from the baking oven by a conveyor belt, if we want to perform a real-time classification of this surface. The basic scheme of the inspection machine is shown in Fig. 1. The inspection machine has two parts.

José A. Gómez Pedrero and Eusebio Bernabeu are with Departamento de Óptica, Universidad Complutense de Madrid, Facultad de Ciencias Físicas, Ciudad Universitaria, s/n, 28040 Madrid, Spain. José A. Peñaranda is with Centro de Estudios e Investigaciones Técnicas de Guipúzcoa, Manuel de Lardiazabal 16, 20009 San Sebastian, Spain.

Received 21 July 1997; revised manuscript received 17 October 1997.

0003-6935/98/101820-06\$15.00/0

(C) 1998 Optical Society of America
First, we have the illumination system, composed of a light source and a lens. This illumination system produces a white-light beam that illuminates the tile surface. The light reflected by the test surface is collected by a CCD color camera connected with a computer. In this way, a color image of the test surface is obtained, and, after this image is processed by the computer, the test surface can be classified.

The CCD camera employed is linear, because (a) a linear camera has more spatial resolution that a matrix one and (b) the transfer time of the image data from the camera to the computer is lower in a linear CCD camera. In these conditions the image of the test surface is acquired line by line when the test surface crosses under the CCD camera object field. Thus the illumination system of the inspection machine must illuminate the object field of the CCD. This implies that the illumination system has to illuminate the narrow rectangular portion of the test surface, which is imaged each time by the CCD camera. In Fig. 1 we can see the coordinate system that we employed. We set the $x$ axis as the conveyor belt displacement direction, the $z$ axis as the normal to the test surface, and finally the $y$ axis as the direction perpendicular to both the $x$ axis and the $z$ axis (see Fig. 1 for details). In this paper we refer to the $x$ axis as the transversal direction and to the $y$ axis as the longitudinal one.

We said above that the classification criteria of the test surfaces are the color and the so-called visual texture. We must describe these criteria in terms of measurable quantities to carry out an effective automatic discrimination of tiles. Two sets of measurable quantities appear. We have those magnitudes that describe the color and those that describe visual texture. We use the relative values of these magni- 


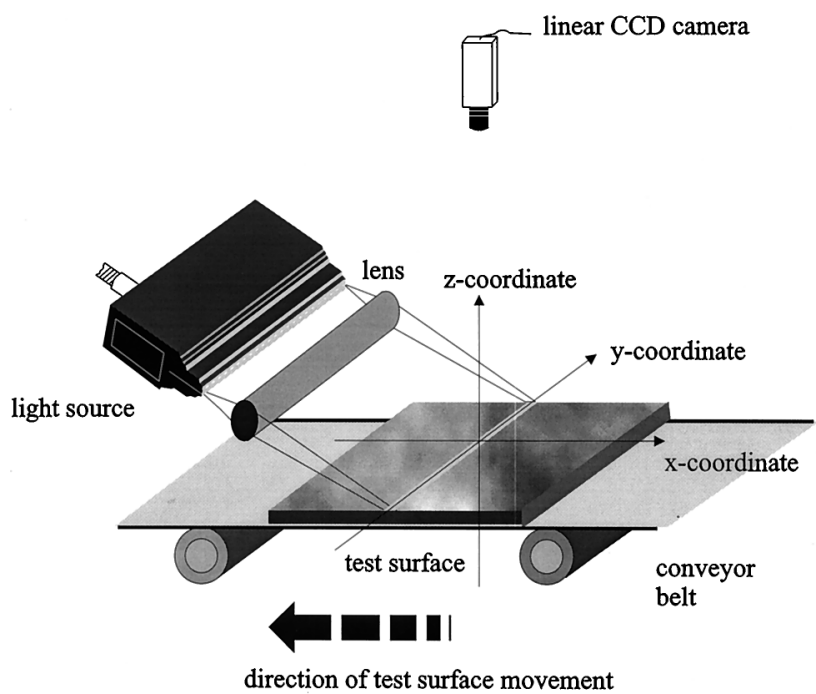

Fig. 1. Experimental setup for the automatic classification of planar surfaces in an industrial production line. We can see the coordinate system.

tudes to discriminate between surfaces. That is, we compare the values of these magnitudes of the test surface with those of a pattern. If the difference between these values is lower than certain limit values, we group the test surface and the pattern in the same set. Otherwise we say that the test surface and the pattern have a different visual appearance.

It is well known that a CCD camera assigns to each image pixel three intensities $\left(I_{R}, I_{G}, I_{B}\right)$ for the red, green, and blue (RGB) channels of the camera. These intensities are related with the spectral irradiance $E(\lambda)$ arriving at the test surface, the spectral reflectance of the surface $\rho(\lambda)$, and with the CCD camera response functions $R_{k}(\lambda)$ of the red, green, and blue channels by means of the following relationship:

$$
I_{k} \propto \int E(\lambda) \rho(\lambda) R_{k}(\lambda) \mathrm{d} \lambda_{k=R, G, B} .
$$

These intensities will be used to characterize the test surface in the following way. Once we know the three intensity values for each image pixel, we calculate the arithmetic mean of these values for all the image pixels, obtaining three mean intensities $\left(\bar{I}_{R}, \bar{I}_{G}\right.$, $\left.\bar{I}_{B}\right)$. Then we can simply subtract these mean intensities with those of the pattern surface. If the relative intensity variations in any red, green, or blue channel are higher than 5\%, we say that the test surface and the pattern have different colors. This can be done as a result of the relationship between the $\left(I_{R}, I_{G}, I_{B}\right)$ intensities and the spectral reflectance of the test surface.

The second classification criterion is the visual texture of the test surfaces. In general the surfaces to be studied present a clear background with a random distribution of dark spots over it. Then we can characterize the visual texture of the surface if we know the mean size of these dark spots. To do this it is necessary to compute three intensity histograms (one for each RGB channel of the CCD). Once we know these histograms we can compute the mean histogram. In these conditions we can describe mathematically the visual texture of the test surface by means of the second-order momentum of the mean histogram:

$$
\mu=\sum_{k=1}^{N}\left(z_{k}-m\right)^{2} h\left(z_{k}\right),
$$

where $N$ is the number of gray levels of the image, $z_{k}$ is the $k$ th gray level, $m$ is the mean gray level, and $h(z)$ is the mean histogram of the test-surface image. The second-order momentum of the mean histogram is closely related to the mean size of the dark spots, if they are randomly distributed over the test surface. ${ }^{1,2}$ An advantage in the use of this operator is the low computational time, which is important in an automatic on-line inspection machine.

From relationship (1) and Eq. (2) we can deduce that there is a strong dependence between the spectral irradiance arriving at the test surface $E(\lambda)$ and both the mean RGB intensities of the test surface $\left(\bar{I}_{R}\right.$, $\left.\bar{I}_{G}, \bar{I}_{B}\right)$ and the second-order momentum of the meanintensity histogram $\mu$. This implies that nondesired variations of $E(\lambda)$ can produce errors in the measurement of $\left(\bar{I}_{R}, \bar{I}_{G}, \bar{I}_{B}\right)$ and $\mu$. Therefore the possibility exists that an incorrect classification of the test surface could be made.

In consequence the illumination system of the inspection machine must be designed to avoid these nondesired spectral irradiance changes. In Section 2 we present the design process of the illumination system. We have found that a system that matches the requirements above is an array of optical fibers illuminated by means of a voltage-stabilized halogen lamp. This array produces a linear light beam collimated by a cylindrical lens. This collimated beam illuminates the object field of the CCD producing a stable spectral irradiance distribution, as the experimental results show. In this way a real-time classification of the test surface can be made.

\section{Analysis of the Illumination System}

As mentioned in Section 1, it is necessary to illuminate the portion of test surface placed at the CCD object field. Because the CCD is linear, its object field is a narrow rectangle. In the particular application of testing ceramic tiles, this rectangle must be $76 \mathrm{~cm}$ long to image the whole width of the conveyor belt (because in general the tile could be rotated with respect to the belt). Thus the primary requirement of the illumination system is to illuminate the rectangular (almost linear) portion of the test surface that coincides with the CCD object field.

To obtain a linear light beam we have used an optical fiber array illuminated by a tungsten halogen lamp supplied by a dc stabilized source. This setup has two advantages: First, it provides a linear white-light beam with a stable spectral radiance (because the voltage of the power supply does not change with the time). Second, the radiance distribution 


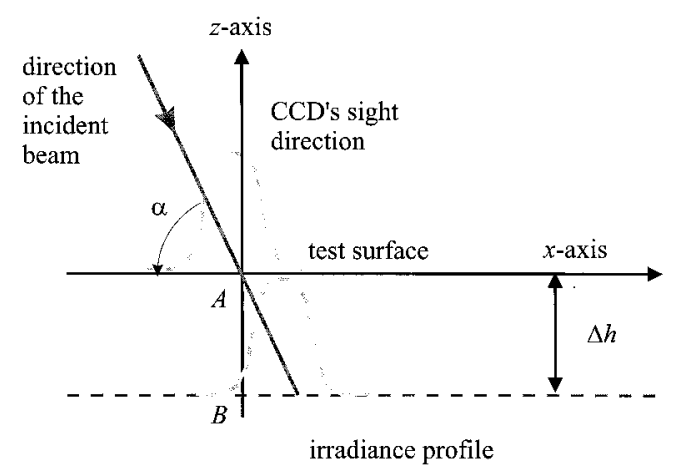

Fig. 2. Effect of vertical surface displacements on the irradiance measured by the CCD camera. Note the irradiance losses due to the horizontal translation of the light profile when the surface is displaced vertically.

along the array direction (the $y$ coordinate) is constant, as shown in Ref. 3 and 4 . We have chosen a commercial system supplied by Fostec. ${ }^{5}$ The propagation direction of the incident beam forms an angle of $\sim 15^{\circ}$ (see Fig. 1) with the plane that contains the test surface. It allows obtaining a maximum amount of reflected light in the normal direction of the test surface.

Once we have a linear light source, we must illuminate the test surface. To do this, the first option is to image the light source over the test surface with a cylindrical lens with the cylinder axis oriented along the array direction. Doing this, we illuminate a narrow rectilinear portion of the test surface, which is imaged by the CCD. This setup guarantees a maximum spectral irradiance at the test surface. However, there is a problem with this setup of the illumination system. This problem appears because the vertical position of the test surface does not remain constant when the surface is carried by the conveyor belt because of the vertical mechanical oscillations of the conveyor belt. These mechanical oscillations are almost inevitable, and consequently there is an error in the vertical position of the test surface. Our measurements have set the numerical value of this error at $\pm 0.5 \mathrm{~mm}$.

The effect of this variation of the test-surface vertical position is illustrated in Fig. 2. In this figure, we present the incident beam reaching the test surface at two different positions of this surface. We also present the projected irradiance transversal profiles of the light beam over the test surface. We can see that in the first position of the test surface the location of the irradiance profile maximum coincides with point $A$ placed at the object field of the CCD camera. But if the surface is displaced vertically $-\Delta h$, the location of the irradiance profile maximum does not coincide with point $B$, placed at the object field of the CCD camera (we suppose that the CCD depth of the field is higher than $\Delta h$ ), because the projected irradiance profile is displaced along the test-surface plane. Because of the nonuniform irradiance profile, the irradiance in $B$ is lower than in $A$, and therefore the irradiance arriving at the CCD sen-

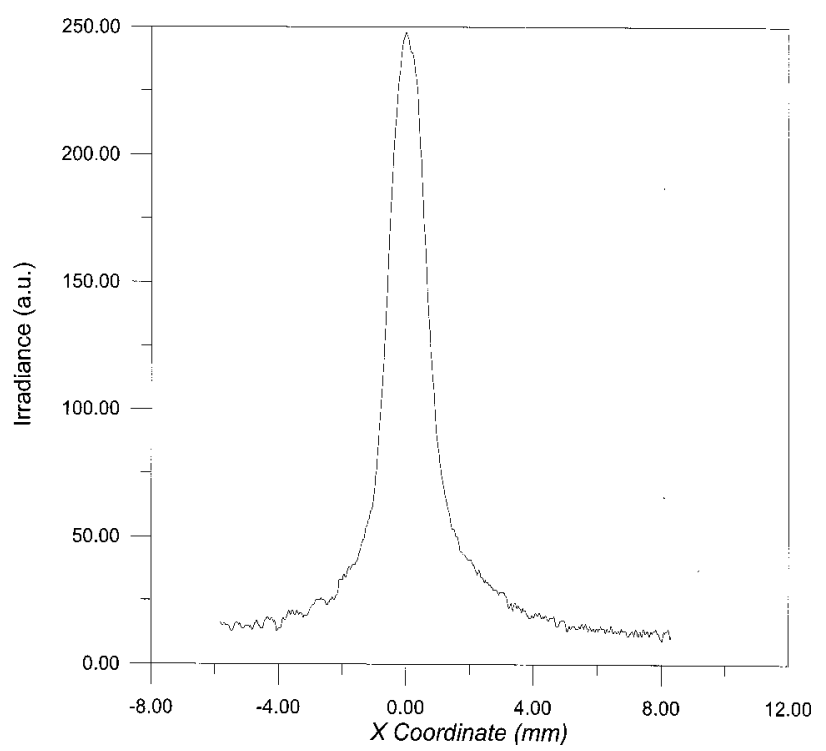

Fig. 3. Irradiance profile at the image plane of the illumination system in the original (commercial) configuration. Note the sharpness of this profile, which must produce great irradiance changes with vertical displacements of the surface.

sor, which is a conjugate of $A$ and $B$, is also lower. Our measurements show that irradiance variation higher than $10 \%$ can produce a classification error of the test surface. ${ }^{1}$ In fact, if the irradiance profile is given by the function $E(x)$ and $E\left(x_{\max }\right)$ is the irradiance arriving at point $A$, the irradiance arriving at point $B$ is given by

$$
E\left[x_{\max }+\Delta h / \tan (\alpha)\right],
$$

where $\alpha$ is defined in Fig. 2. On the other hand, we define $\omega$ as follows:

$$
E\left(x_{\max }+\omega\right)=0.1 E\left(x_{\max }\right) .
$$

From this definition, it is clear that, if $\omega<|\Delta h| /$ $\tan (\alpha)$, the inspection machine is sensitive to testsurface oscillations. More accurately, if $\Delta h= \pm 0.5$ $\mathrm{mm}$ and $\alpha=15^{\circ}$, then $\omega$ should be higher than 1.86 $\mathrm{mm}$. This implies that we must illuminate the tile with a wide light beam to ensure that the inspection machine is not sensitive to the mechanical oscillations of the test surface.

We can see in Fig. 3 the appearance of the transversal irradiance profile at the test surface when the illuminating system forms an image of the fiber array over the test surface (placed $21 \mathrm{~cm}$ from the fiber array, which is the optimum distance between the light source and the test surface). This profile was obtained by placing at the image plane of the illuminating system a translucent diffuser screen (made by flat optical glass with magnesium oxide deposed over it); behind this screen a CCD matrix camera forms the image of the irradiance distribution at the diffuser screen. The irradiance profile shown in Fig. 3 is very sharp, as could be expected. The value for $\omega$ is $0.25 \mathrm{~mm}$, which is much lower than $1.86 \mathrm{~mm}$. It is obvious that we must find another setup for the 
(a)
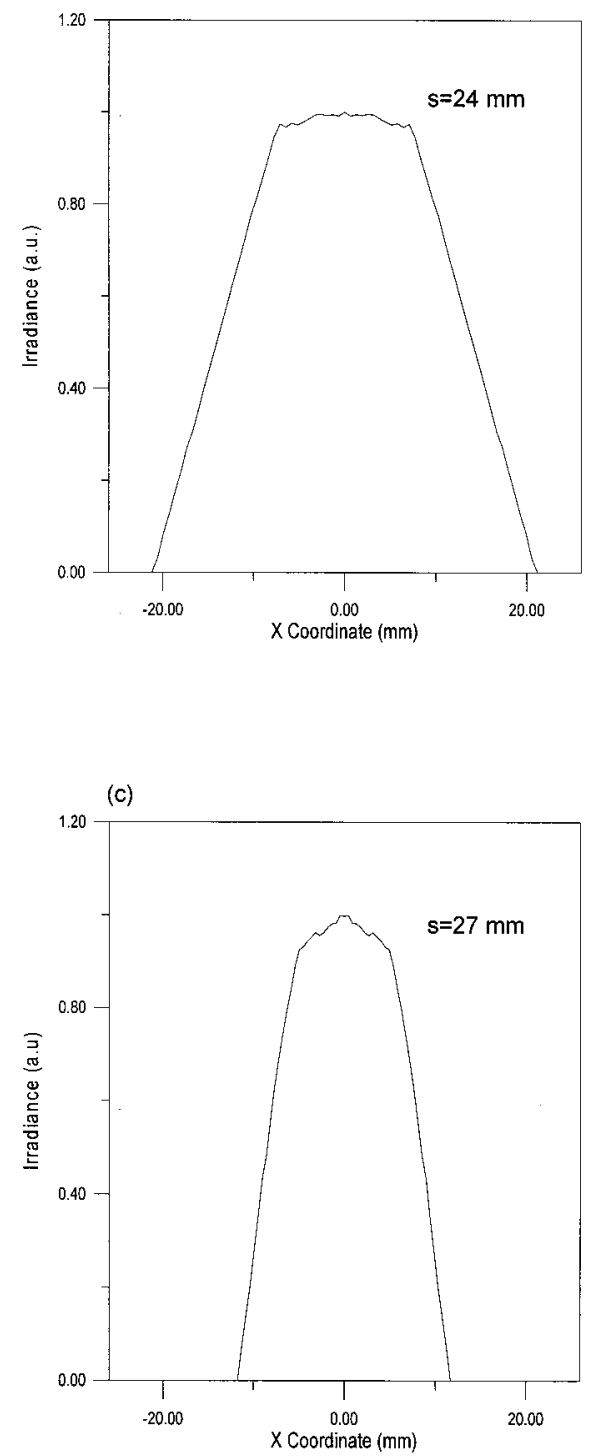
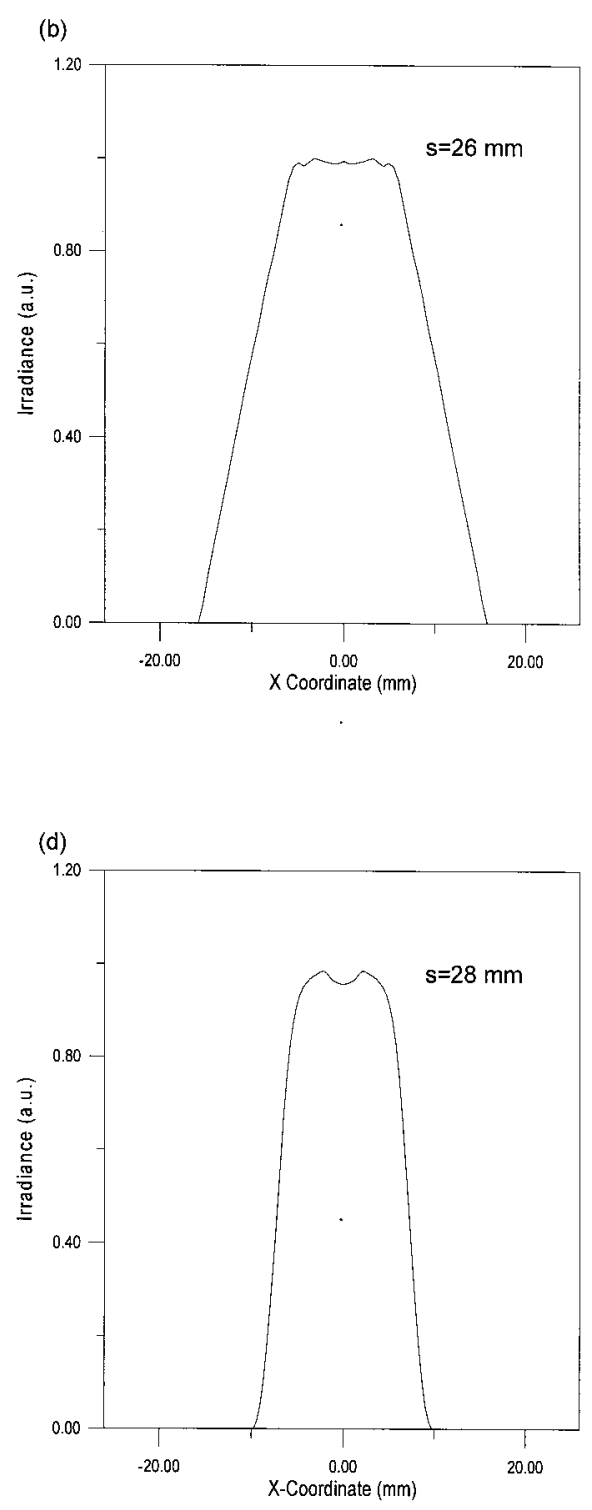

Fig. 4. Irradiance profiles of the light beam at a planar screen, obtained by numerical simulation for several distances between the light source and the lens: (a) 24 $\mathrm{mm}$, which corresponds to a collimated beam; (b) $26 \mathrm{~mm}$; (c) 27 $\mathrm{mm}$; (d) $28 \mathrm{~mm}$. For (b), (c), and (d), a converging beam reaches the screen. illumination system if we want to avoid the effect of the mechanical oscillations of the test surface in the reflected irradiance.

In general, we can illuminate the test surface in four different ways. First, we can form the image of the light source on the test surface. As we have already seen, this solution cannot be applied to this case because of the vertical displacements of the test surface. Second, we can illuminate the image with a convergent beam, placing, for example, the test surface between the lens and the image of the source. Third, we can illuminate the test surface with a divergent beam. This solution is not acceptable because of the low values of the irradiance at the test surface that we obtained with this setup. Fourth, we can illuminate the test surface with a collimated beam. It is obvious that we must discriminate between the convergent and the collimated beams.

To carry out this discrimination, we made a numerical simulation of the illumination system with
Matlab. In concrete, we have calculated the irradiance transversal profiles of the light beam at the test surface for four relative positions between the light source and the cylindrical lens but with a constant distance between the light source and the test surface of $21 \mathrm{~cm}$. The calculus procedure employed has been the usual method of calculating a large number of light rays that exit the source and arrive at the screen, obtaining the corresponding spot diagram over the screen and calculating the histogram of these spot diagrams (which is proportional to the irradiance at the screen). The range of distances between the lens and the light source comprises the focal length of the lens (corresponding to the collimated beam) and $95 \%$ of the object distance at which the lens forms the image of the source over the test surface. For all these object distances the screen is placed before the image of the source, so a converging beam is incident on the screen. As we can see in the figure (see Fig. 4), the best profile is that correspond- 

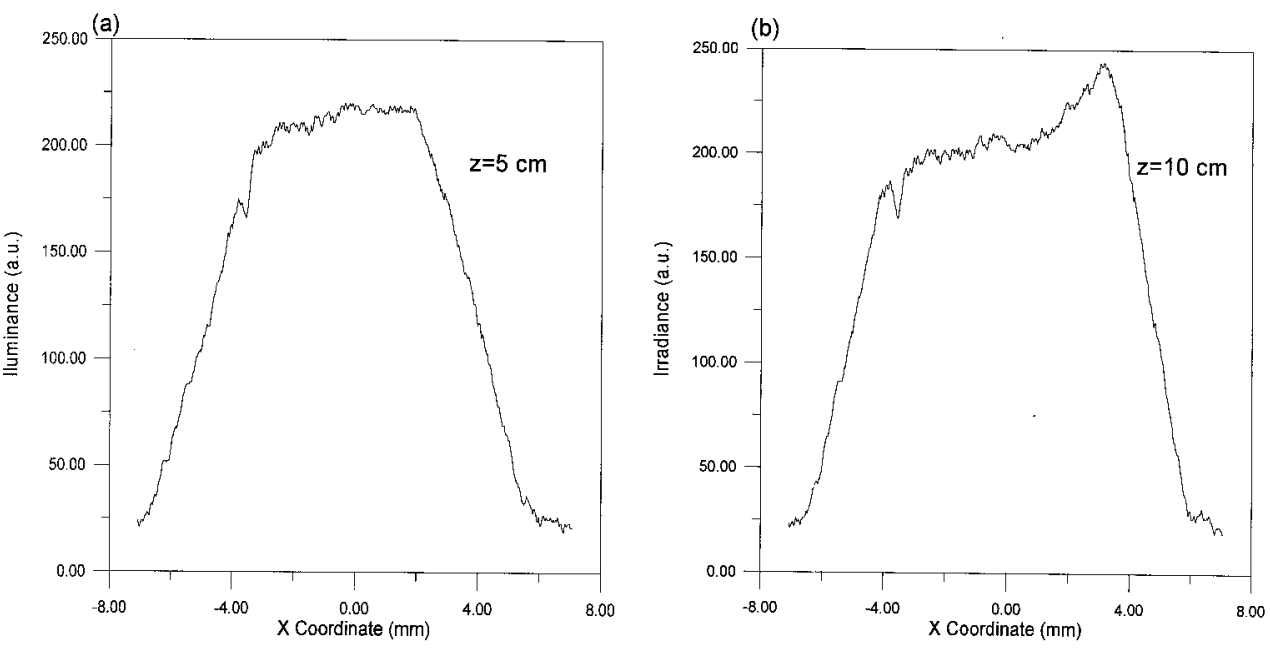

Fig. 5. Experimental irradiance profiles of the collimated beam for several distances between the
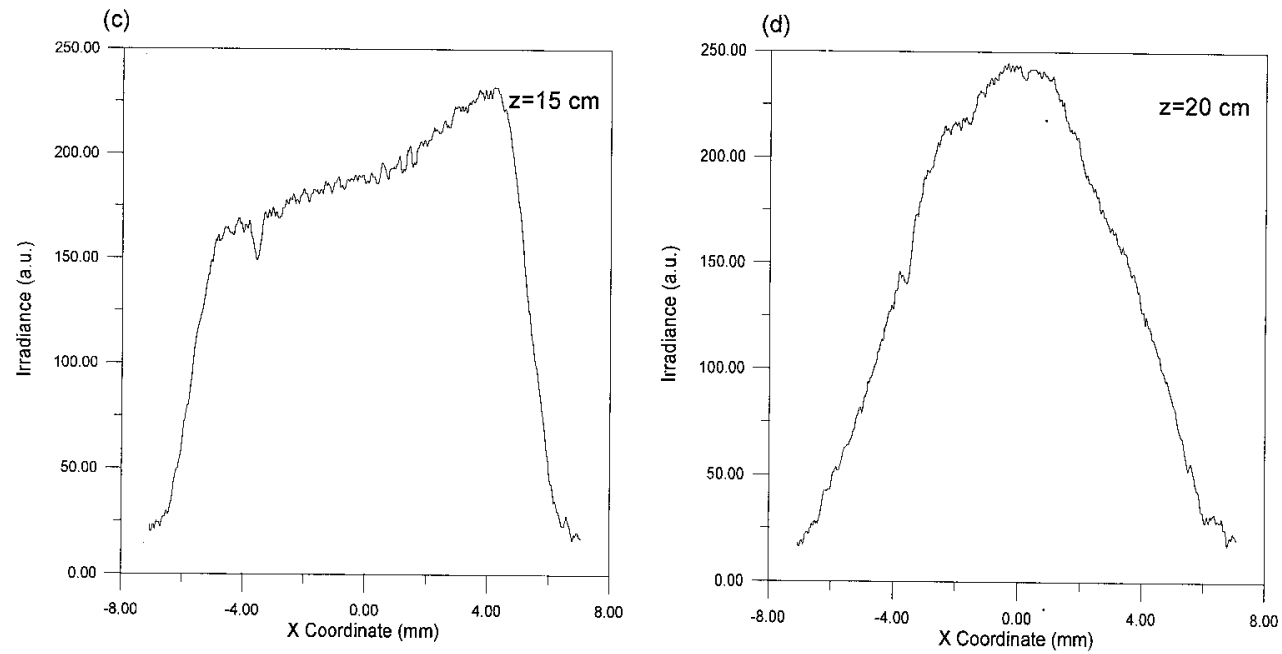
screen and the lens.

ing to the collimated beam, because it is the wider one. Thus we propose the use of collimated illumination for the inspection machine.

\section{Experimental Results}

As we have seen in Section 3, a variation of the irradiance arriving at the linear CCD with vertical displacements of the test surface is not expected whether or not we illuminate the test surface with a collimated beam. To prove this we have measured the irradiance profiles of the illuminated beam at several distances from the lens, using the same experimental setup described above (see Section 2) but with the light source placed at the focal plane of the cylindrical lens. In Fig. 5 we have plotted the irradiance profiles measured in planes located 5, 10, 15, and $20 \mathrm{~cm}$ away from the collimating lens. We can see that the profiles plotted in (a) and (b) show low irradiance variations at their center. However, the profiles in (c) and (d) do not present the same flat-top shape. This is due to the vignetting effect that suffers the light beam. It means that the range of dis- tances between the optical system and the test surface is restricted.

On the other hand, we have calculated the irradiance values at the central point of the profiles shown in Fig. 5, showing the results in Table 1. In this table, we can also find the difference in the central point irradiance between two consecutive profiles and the values of $\omega$. We have obtained values of $\omega$ greater than $2.61 \mathrm{~mm}$. This means that effectively the use of collimated illumination makes the process of classification of the planar surface with our system independent of low mechanical oscillations of the test surface.

Table 1. Collimated Beam Characteristics

\begin{tabular}{rccc}
\hline$z(\mathrm{~cm})$ & $E$ (a.u.) & $\Delta E$ (a.u.) & $\omega(\mathrm{mm})$ \\
\hline 5 & 184 & - & 2.95 \\
10 & 210 & 26 & 3.26 \\
15 & 220 & 10 & 3.43 \\
20 & 223 & 3 & 2.61 \\
\hline
\end{tabular}


According to these results, we have used collimating illumination in the industrial prototype of the test machine, finding not measurable variations of the irradiance collected by the CCD array, with vertical surface displacements of $1 \mathrm{~mm}$. We must point out that the total irradiance arriving at the test surface is lower when we use a collimated beam instead of forming the image of the light source over the test surface. This problem has been solved, increasing the radiance of the light source.

\section{Conclusions}

We have designed the illuminating system of an apparatus for automatic classification of the planar surface in a production line. We have found an optimum solution consisting of a linear array of optical fibers illuminated by means of a halogen lamp and a cylindrical collimating lens. This setup produces a linear collimated beam, which illuminates the test surface, avoiding undesired changes in the irradiance that arrives at the test surface. The use of a collimated beam has made the test system robust to vertical oscillations of the test surface, as shown in both the numerical simulation and the experimental results presented in this paper. We must say that the illumination system designed by us has been applied successfully to the industrial prototype of the floor tiles inspection machine.

However, we must point out that the use of collimated illumination is not the only solution, and it would be desirable to find another solution that could lower the irradiance losses, which implies the use of collimated illumination without increasing the radiance of the light source. Perhaps a solution could be use of a traditional projector system, such as a Köhler one, but we were not able to obtain a stable light beam with this kind of system because of alignment problems related to the extremely long dimensions of the cylindrical lens employed $(75 \mathrm{~cm})$.

We thank Agustín González-Cano, Juan C. Martínez-Anton, and José Alonso for kind and useful comments. Also we thank Manuel Fuentes, Centro de Estudios e Investigaciones Técnicas, for concern for the project and kindness.

\section{References}

1. J. A. Peñaranda, "Sistema de visión artificial para la clasificación 'on-line' por color y textura de piezas de 'Gres porcelanico'," Ph.D. thesis (Universidad de Navarra, San Sebastian, Spain, 1997).

2. J. A. Peñaranda, A. P. Pobil, and M. A. Serna, "Classification system for pieces of Porcelanatto based on computer vision," in Automated $3 D$ and $2 D$ Vision, R. Ahlers, D. W. Braggins, and G. W. Kamerman, eds., Proc. SPIE 2249, 339-348 (1994).

3. J. D. Rees and W. Lama, "Some radiometric properties of gradient-index fiber lenses," Appl. Opt. 19, 1065-1069 (1990).

4. M. Kawazu and Y. Ogura, "Application of gradient-index fiber arrays to copying machines," Appl. Opt. 19, 1105-1112 (1980).

5. Product Bulletin 4001 (Fostec Inc., 4950-C, Eisenhower Avenue, Alexandria, Va., 1995). 